\title{
Sorafenib tosylate for advanced kidney cancer: lucky loser and magic box at the same time
}

\author{
Camillo Porta
}

Published online: 10 August 2011

(C) Springer-Verlag 2011

Despite being the very first molecular targeted agents registered for the treatment of advanced kidney cancer (in the post-cytokines setting) [1], Sorafenib seemed to lose its momentum as a credible first-line treatment option, when it proved not to be superior to Interferon within a randomized phase II trial [2].

Even though the results of that trial have been highly criticized-mainly due to patient selection bias-these negative results, together with the concomitant availability of other highly active treatments, such as Sunitinib, the combination of Bevacizumab plus Interferon, Temsirolimus (for poor risk patients) and, more recently, also Pazopanib, greatly limited its front-line use almost only to those few patients still treated first-line with cytokines, or to ill-defined, special situations (e.g., elderly patients, patients with cardiovascular co-morbidities, etc.) [3].

As far as second-line, where its use is supported by almost only retrospective, studies [4], the publication of the randomized controlled, phase III trial of Everolimus [5] seemed to have moved Sorafenib back to third, or even subsequent, line.

Recent data-surprisingly gathered from non-Bayersponsored trials-seem to have someway revitalized this drug in its very first oncological indication, i.e., kidney cancer.

Indeed, in the recently presented first-line AMG 386 trial [6], Sorafenib — which was used as a control arm-

\section{Porta $(\bowtie)$}

Medical Oncology, IRCCS San Matteo University Hospital

Foundation, Piazzale C. Golgi 19, 27100 Pavia, Italy

e-mail: c.porta@smatteo.pv.it

C. Porta

Gruppo Italiano di Oncologia Nefrologica

(GION, Italian Nephro-Oncology Group), Pavia, Italy yielded a progression-free survival (PFS) of 9.0 months, almost doubling the PFS reported in the first-line trial against Interferon [2], moving Sorafenib closer to the other treatment options commonly used in first-line treatment options that, as in the case of Sunitinib, saw their performance someway resized when tested, similar to the two above-mentioned Sorafenib studies, within smaller (and therefore more biased), randomized phase II studies [7].

Similarly, when the results of the Axitinib AXIS trial were disclosed [8], Sorafenib gained further momentum also in the second-line setting; how is it possible, given that Sorafenib was the loser against Axitinib?

It happened that Sorafenib, given after a first-line treatment (mainly Sunitinib), yielded a PFS that was almost superimposable to the one observed for Everolimus after Sunitinib.

Even though it should be noted that, in the vast majority of cases, Everolimus-treated patients were not pure secondline patients, the results achieved with Sorafenib in the AXIS trial do support the option of its use in a second-line setting, as a partial alternative to Everolimus, at least until Axitinib registration.

Indeed, the level of evidence of the two putative options (i.e., Sorafenib and Everolimus) is now the same.

At this point, second-line treatment should be tailored on a patient-to-patient basis, taking into account patients' characteristics (especially co-morbidities), disease characteristics, as well as treatment aim [9].

Furthermore, a recent report from a Japanese group [10] clearly suggests that the concept of re-challenge may also apply to Sorafenib, and not just to Sunitinib [11].

Indeed, the authors showed-even though in a retrospective way - that clinical benefit may be achieved with Sorafenib also in patients already treated with this same multikinase inhibitor [10]. 
Even though, for sure, the lack of alternative treatment options is definitely not an issue in kidney cancer nowadays, Sorafenib (and Sunitinib) re-challenge could be a reasonable treatment option where alternative therapies are not available (and where regulatory authorities do allow it).

More interestingly, both the prospective AXIS data, as well the retrospective Japanese report do suggest that a continuous inhibition of the VEGFR(s) pathway could be of benefit for advanced kidney cancer patients.

The activity of the mTOR inhibitor Everolimus in patients failing a first or second-line treatment with an antiVEGF pathway tyrosine kinase inhibitor do not necessarily contradict this hypothesis. Indeed, due to the peculiar molecular pathogenesis of kidney cancer-so dependent on angiogenesis, due to the frequent mutation of the VHL gene also in sporadic cases [12] - it is also possible that presently available mTOR inhibitors also (which indeed are just TORC1 complex inhibitors) could be active due to their indirect inhibition of angiogenesis [13].

As a whole, it is clear that we still have a lot to know about the presently available treatments for kidney cancer (Sorafenib in primis), and that the phase III trials performed to date simply cannot address all the issues still on the table.

Finally, I cannot but agree with the strong statement made by Chris Ryan during the educational session at the 2010 annual meeting of the American Society of Medical Oncology (ASCO), when he provocatively proposed his personal (and simplified) algorithm with the strong sentence: choose any agent You want. Use it well.

Conflict of interest $\mathrm{CP}$ acted as a paid speaker or consultant for Bayer Schering Pharma, Pfizer Oncology, Hoffman La Roche, GSK and Novartis Pharma; he also received research grants from Bayer Schering Pharma and Novartis Pharma.

\section{References}

1. Escudier B, Eisen T, Stadler WM et al (2007) Sorafenib in advanced clear-cell renal-cell carcinoma. N Engl J Med 356:125-134
2. Escudier B, Szczylik C, Hutson TE et al (2009) Randomized phase II trial of first-line treatment with sorafenib versus interferon Alfa-2a in patients with metastatic renal cell carcinoma. J Clin Oncol 27:1280-1289

3. Bellmunt J, Fishman M, Eisen T, Quinn D (2010) Expert opinion on the use of first-line sorafenib in selected metastatic renal cell carcinoma patients. Expert Rev Anticancer Ther 10:825-835

4. Porta C, Paglino C, Imarisio I (2011) Sequencing tyrosine kinase inhibitors or immediately switching to mTOR inhibitors in advanced kidney cancer: a critical review. Eur J Med Clin Oncol 2:67-72

5. Motzer RJ, Escudier B, Oudard S et al (2008) Efficacy of everolimus in advanced renal cell carcinoma: a double-blind, randomised, placebo-controlled phase III trial. Lancet 372:449-456

6. Rini BI, Szczylik C, Tannir NM, et al (2011) AMG 386 in combination with sorafenib in patients (pts) with metastatic renal cell cancer (mRCC): a randomized, double-blind, placebo-controlled, phase II study. J Clin Oncol 29 (suppl. 7; abstr. 309)

7. Motzer RJ, Hutson TE, Olsen MR, et al (2011) Randomized phase II multicenter study of the efficacy and safety of sunitinib on the $4 / 2$ versus continuous dosing schedule as first-line therapy of metastatic renal cell carcinoma: renal EFFECT Trial. J Clin Oncol 29 (suppl. 7; abstr. LBA308)

8. Rini BI, Escudier B, Tomczak P, et al (2011) Axitinib versus sorafenib as second-line therapy for metastatic renal cell carcinoma (mRCC): Results of phase III AXIS trial. J Clin Oncol 29 (suppl.; abstr. 4503)

9. Porta C, Bellmunt J, Eisen T, Szczylik C, Mulders P (2010) Treating the individual: The need for a patient-focused approach to the management of renal cell carcinoma. Cancer Treat Rev $36: 16-23$

10. Nozawa M, Matsumura N, Yasuda M, et al (2011) Activity of retreatment with sorafenib for metastatic renal cell carcinoma. J Clin Oncol 29 (suppl. 7; abstr. 404)

11. Zama IN, Hutson TE, Elson P et al (2010) Sunitinib rechallenge in metastatic renal cell carcinoma patients. Cancer 116: $5400-5406$

12. Clark PE (2009) The role of VHL in clear-cell renal cell carcinoma and its relation to targeted therapy. Kidney Int 76:939-945

13. Mulders P (2009) Vascular endothelial growth factor and mTOR pathways in renal cell carcinoma: differences and synergies of two targeted mechanisms. BJU Int 104:1585-1589 